(C) Masson, Paris, 1980.

\title{
Helminthes du Renard commun (Vulpes vulpes L.) dans la région du Massif Central (France).
}

\author{
par A.-F. PETAVY * et S. DEBLOCK ** \\ " C.H.U. de Clermont-Ferrand, Service de Parasitologie ( $P^{r}$ Coutet), \\ B.P. 69, F 63003 Clermont-Ferrand. \\ ** Faculté de Pharmacie, rue de Pr Laguesse, F 59045 Lille Cedex.
}

RESUME. Soixante-neuf renards sauvages capturés en Auvergne de janvier 1977 à janvier 1978 présentent des helminthes intestinaux dans la proportion de $76,8 \%$. $59,4 \%$ hébergent des Cestodes, $72,4 \%$ des Nématodes et aucun des Trématodes. La liste des espèces identifiées est la suivante: $1^{\circ}$ Cestodes. A) Formes adultes fertiles de Mesocestoides litteratus chez 27,5 \% des hôtes; de Taenia crassiceps $(15,9 \%)$; de Taenia polyacantha $(27,5 \%)$; de Echinococcus multilocularis $(7,2 \%)$; de Anomotaenia paradoxa $(4,34 \%)$. B) Formes immatures de Taenia pisiformis $(2,89 \%)$; de $T$. ovis $(1,44 \%)$ et d'un Cestode sp. $(1,44 \%) .2^{\circ}$ Nématodes. Uncinaria stenocephala chez 68,1 \% des hôtes ; Ancylostoma caninum (1,4\%); Molineus patens $(1,4 \%)$; Oxyuridé sp. $(1,44 \%)$; Toxocara canis $(27,5 \%)$; Toxascaris leonina $(33,3 \%)$. Les espèces d'helminthes identifiées dans le centre de la France ne se différencient pas des espèces signalées en Europe chez le même hôte, à l'exception de A. paradoxa, Dilépididé d'Oiseau, tout à fait inhabituel chez un Carnivore.

\section{Helminths of the common fox (Vulpes vulpes L.) from the Massif Central (France).}

SUMMARY. Sixty nine wild foxes originating from Auvergne for the period January 1977 to January 1978 have shown intestinal parasites in the proportion of 76,8 per cent. 59,4 per cent are invaded with Cestodes; 72,4 per cent with Nematodes and none with Trematodes. The list of the identified species is following: $1^{\circ}$ Cestodes. A) Adults and fertile forms of: Mesocestoides litteratus in 27,5 per cent of the hosts; Taenia crassiceps in 15,9 p.c.; T. polyacantha in 27,5 p.c; Echinococcus multilocularis

Accepté le 30 janvier 1980. 
in 7,2 p.c; Amoebotaenia paradoxa in 4,3 p.c. B) Immature forms of: Taenia pisiformis in 2,89 p.c.; T. ovis in 1,44 p.c. $2^{\circ}$ Nematodes. Uncinaria stenocephala in 68,1 per cent of the hosts; Ancylostoma caninum in 1,4 p.c.; Molineus patens in 1,4 p.c.; Oxyurid sp. in 1,4 p.c.; Toxocara canis in 27,5 p.c. and Toxascaris leonina in 33,3 p.c. The composition of the helminth fauna of the foxes in the center countries of France is not different of the European species from the same host, except Amoebotaenia paradoxa, Dilepidid cestode of birds, quite unusual from a carnivorus mammal.

\section{Introduction}

La description clinique de deux cas humains d'échinococcose alvéolaire du foie originaires de la région du Massif Central en France par Rey et coll., 1977, était l'occasion de rechercher l'existence du tænia multiloculaire adulte chez les hôtes définitifs naturels fréquentant la même région géographique que les malades (1). La nomenclature et la description de la faune helminthologique du contenu intestinal de renards adultes sauvages capturés dans les départements du Puy-de-Dôme et du Cantal, ainsi que dans les zones limitrophes des départements adjacents (Creuse et Allier), font l'objet de notre étude. Celle-ci acquiert un surcroît d'intérêt du fait du nombre restreint d'enquêtes similaires menées jusqu'à présent sur le territoire national (voir Coudert et coll., 1970), en dépit de la chasse active menée contre le renard en raison de l'épizootie de rage qui le décime depuis plusieurs années.

\section{Matériel et méthodes}

Soixante-neuf renards (Vulpes vulpes L.) ont fait l'objet de nos investigations ; 43 proviennent du département du Puy-de-Dôme, 18 du Cantal, 6 de 1'Allier et 2 de la Creuse (fig. 1). Ils ont été capturés par un personnel spécialisé de janvier 1977 à janvier 1978. Immédiatement après l'abattage, chaque animal a reçu des injections intraabdominales de formol à $10 \%$; puis, après l'avoir enfermé dans un sac de plastique épais, on l'expédiait dans les délais les plus rapides au laboratoire de parasitologie de Clermont-Ferrand, accompagné d'une fiche signalétique mentionnant lieu et date de capture, âge et sexe. Les helminthes furent prélevés lors de l'autopsie au fur et à mesure de l'ouverture du tube digestif par segments d'une dizaine de centimètres.

(1) Un troisième cas humain, actuellement en évolution. a été diagnostiqué ultérieurement dans la même contrée (Cantal). Un quatrième cas, fatal, a été diagnostiqué dans la région parisienne, avec de fortes probabilités d'infestation du malade dans le département de la Creuse (in Bourée et coll., 1978. - Nouv. Presse Méd., 7, [28] : 2 480). 
et plongés dans l'eau formolée afin de parfaire leur fixation. Les cestodes furent colorés au trichrome de Wheatley ou aux carmins chlorhydrique ou acétique selon le cas, puis montés au baume, in toto ou après débitage histologique par la méthode classique à la paraffine. Les Nématodes furent déterminés individuellement dans des liquides de montage extemporané (eau, lactophénol), éventuellement après dissection partielle. ou section transversale des extrémités convenabies.

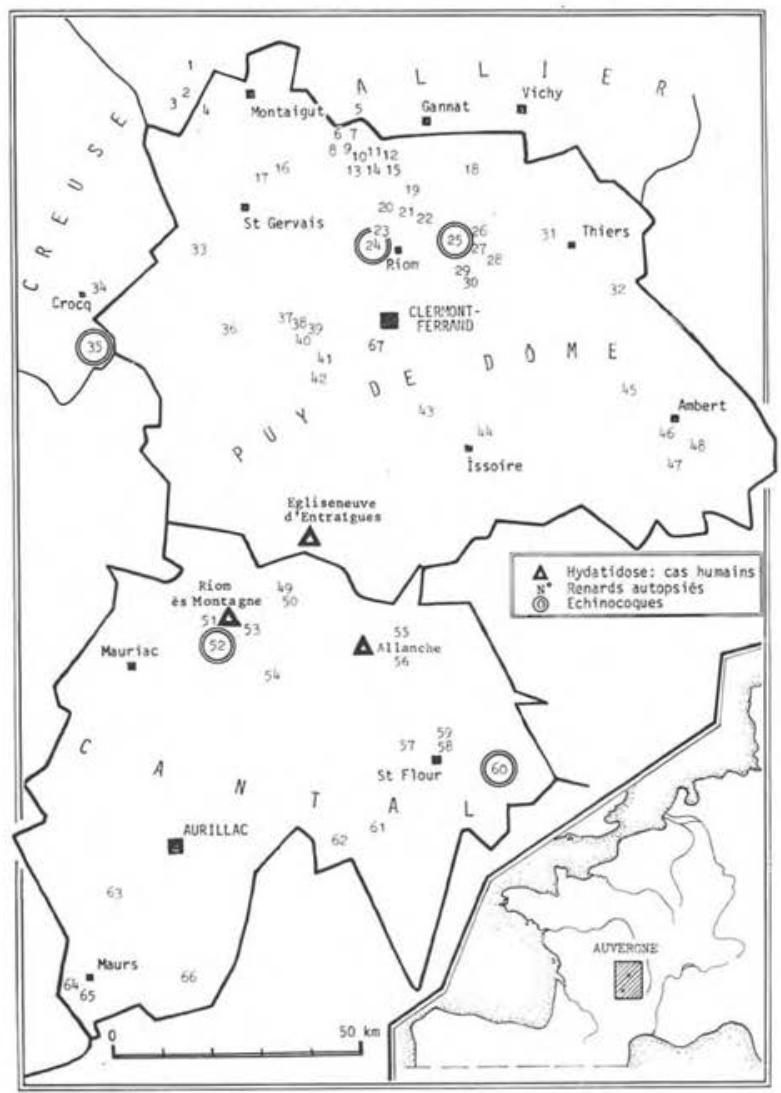

Fig. 1. Répartition géographique des renards autopsiés. Chaque numéro de 1 à 69 correspond à un animal. Les numéros cerclés correspondent à un renard parasité par des E. multilocularis.

\section{Résultats des autopsies \\ I. - CESTODES}

Synopsis des espèces rencontrées.

I. - Famille des Mesocestoididae Perrier, 1897. Genre Mesocestoides Vaillant, 1863 : Mesocestoides litteratus (Batsch, 1786). 
II. - Famille des Taeniidae Ludwig, 1886.

1) Genre Taenia Linné, 1758 : Taenia crassiceps (Zeder, 1800), Rud., 1810. T. polyacantha Leuckart, 1856. Formes immatures de T. pisiformis (Bloch, 1780), T. ovis (Cobbold, 1869) et d'un Taenia sp.

2) Genre Echinococcus Rudolphi, 1801: E. multilocularis Leuckart, 1863.

III. - Famille des Dilepididae Railliet et Henry, 1909. Genre Amoebotaenia Cohn, 1900 : A. paradoxa (Rud., 1802).

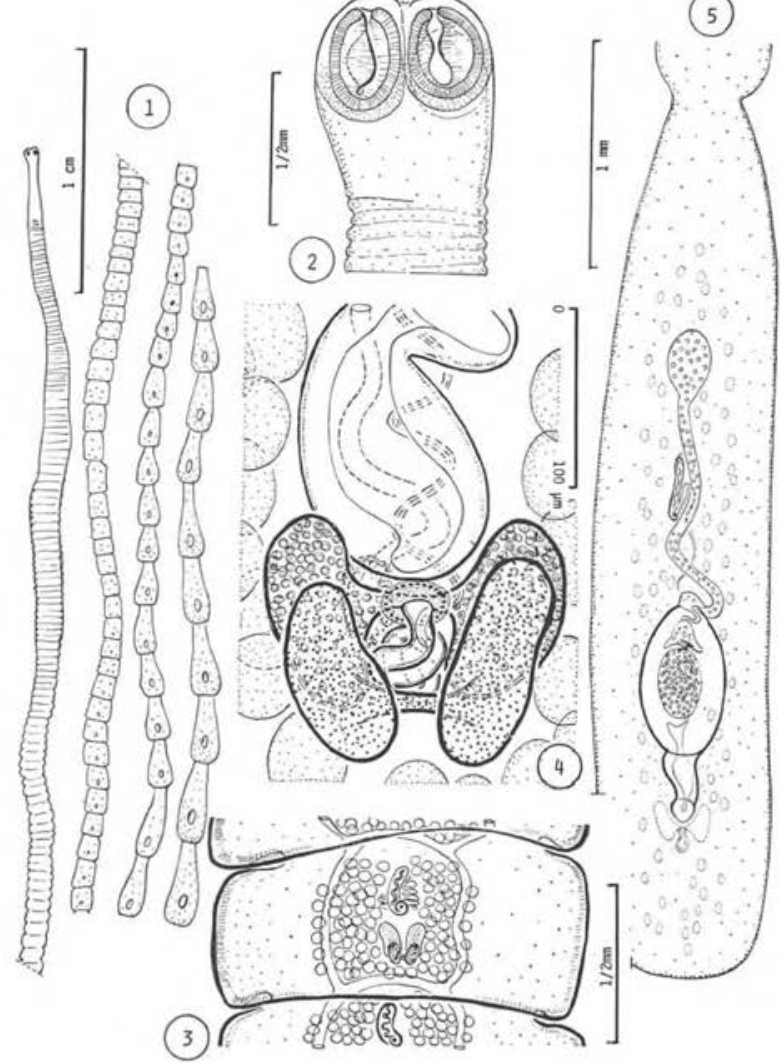

Fig. 2. Mesocestoides litteratus. 1. Strobile complet. 2. Scolex. 3. Anneau mûr. 4. Appareil reproducteur femelle. Vitellogène supère (en pointillés). Ovaire dessous, avec l'oviducte contourné passant en position infère, où se situe la glande de Mehlis. Le fin canal vaginal et l'utérus sinuent sous l'organe parutérin ovoïde (figuré en clair). Testicules périphériques. 5. Anneau gravide terminal. 
Mesocestoides litteratus. 19 hôtes parasités, soit 27,5 \% (fig. 2).

Le nombre des parasites par hôte varie de un à quelques dizaines.

Discussion. Le strobile mûr est toujours trop court (inférieur à $150 \mathrm{~mm}$ ) pour que l'espèce puisse appartenir à l'espèce lineatus qui atteint 300 à $2500 \mathrm{~mm}$. Il s'agit donc vraisemblablement de l'espèce $M$. litteratus (Batsch). L'hôte définitif s'infeste par l'ingestion de tétrathyridiums, larves parenchymateuses parasites de divers vertébrés à sang froid ou chaud, situées à l'état libre ou enkysté dans des régions diverses de leur organisme. L'espèce est signalée chez les renards européens.

Taenia crassiceps. 12 hôtes parasités, soit 17,39 \% (fig. 3).

Le nombre des parasites par hôte est généralement peu élevé.

La larve vésiculeuse correspond au Cysticercus longicollis de Rudolphi; elle se loge généralement dans le conjonctif sous-cutané de divers petits rongeurs. Le contenu intestinal d'un renard a révélé des cysticerques au scolex non encore évaginé dont les crochets ont été rapportés à ceux de l'espèce crassiceps par leur taille (180-185 $\mu \mathrm{m}$ et $138 \mu \mathrm{m}$ ), leur forme et leur nombre. T. crassiceps est signalé chez les renards d'Europe ; en fait, l'espèce existe dans tout l'hémisphère boréal.
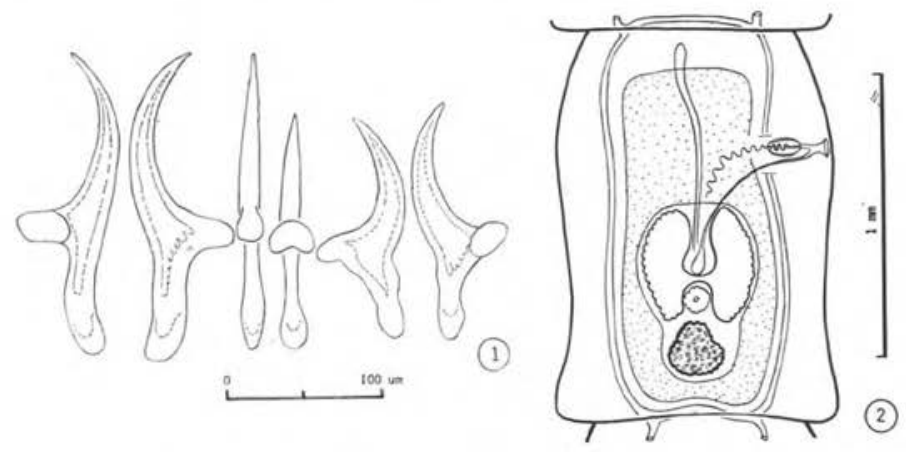

Fig. 3. Taenia crassiceps. 1. Crochets du rostre (face et profil). 2. Diagramme de l'anneau mûr. En pointillés, l'extension du champ testiculaire. Vitellogène massif, entouré de testicules.

Taenia polyacantha. 19 hôtes parasités, soit 27,53 \% (fig. 4).

Le nombre des parasites par hôte est très variable; toutefois le nombre des cestodes immatures est toujours nettement plus élevé que celui des cestodes mûrs. Sept hôtes seulement hébergent des strobiles complets.

La larve parenchymateuse apparentée aux cysticercoïdes est dite "tétrathyridium "; elle se situe à l'état libre dans les cavités abdominales ou thoraciques de nombreux rongeurs Muridés et Cricétidés. Taenia polyacantha est signalé chez les renards d'Europe et d'Asie. 


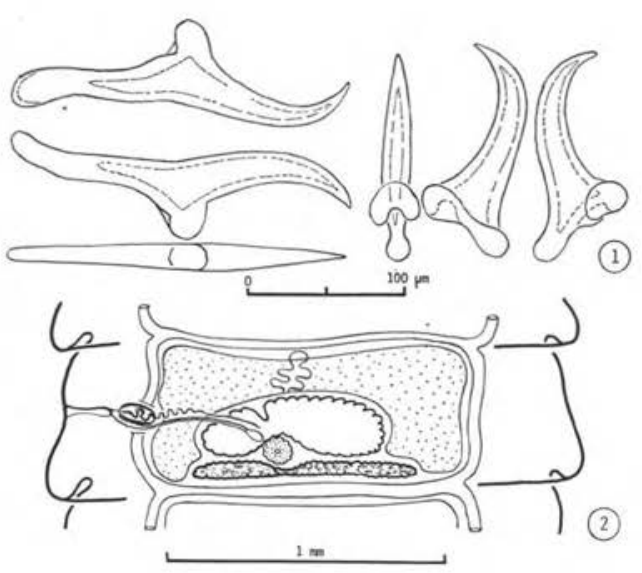

Fig. 4. Taenia polyacantha. 1. Crochets du rostre (face et profil). 2. Diagramme de l'anneau mûr. En pointillés, l'extension du champ testiculaire. Vitellogène linéaire, non entouré de testicules.

Remarques: Les contenus intestinaux ont révélé par ailleurs:

1. chez deux renards, des scolex non strobilisés porteurs de deux couronnes de 20-22 crochets chacune mesurant 245 à $265 \mu \mathrm{m}$ et 145 à $150 \mu \mathrm{m}$ de long. La taille, la forme et le nombre des crochets peuvent faire supposer qu'il s'agit de cysticercoïdes récemment ingérés de Taenia pisiformis (fig. 5) ;

2. chez un renard, quelques scolex ayant bourgeonné une faible longueur de cou. Ils portent deux couronnes de 18 crochets chacune, mesurant 167 à $180 \mu \mathrm{m}$ et 120 à $125 \mu \mathrm{m}$. La taille, la forme et le nombre des crochets peuvent faire supposer qu'il s'agit de scolex de Taenia ovis (fig. 5);

3. chez un autre renard, un scolex non strobilisé porteur de deux couronnes de 34 crochets de $170-200 \mu \mathrm{m}$ et 145 à $155 \mu \mathrm{m}$; il ne peut être identifié ;

4. chez un dernier renard, trois scolex non strobilisés porteurs de deux couronnes de 19 crochets chacune mesurant respectivement 235 à $240 \mu \mathrm{m}$ et $140 \mu \mathrm{m}$ de long. Leur forme prête à supposer qu'il s'agit peut-être d'une limite inférieure de taille des crochets de l'espèce pisiformis (fig. 5).

Echinococcus multilocularis. 5 hôtes parasités, soit 7,2 \% (fig. 6).

Le nombre des parasites par hôte est respectivement de 3,10, 27, plusieurs centaines, le dernier en hébergeant un nombre indéterminé mais peu élevé. 


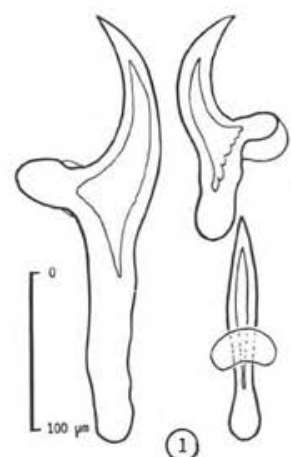

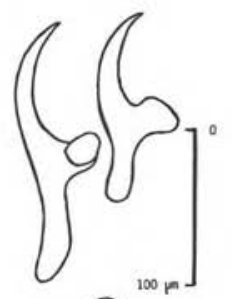

(2)

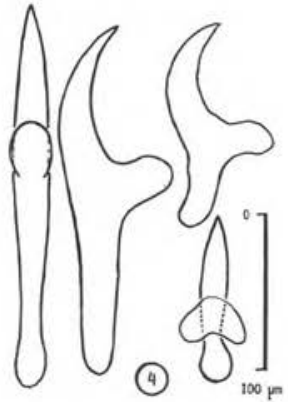

Fig. 5. 1. Crochets du rostre de $T$. pisiformis. 2. Crochets du rostre de $T$. ovis. 3. Crochets du rostre d'un ténia sp. 4 . id.

L'espèce se présente sous la forme de strobiles longs de 900 à $1750 \mu \mathrm{m}$ comportant 2 ou 3 segments issus du scolex prolongé du cou. Le scolex mesure 145-190 $\mu \mathrm{m}$ de diamètre et le rostre $80-90 \mu \mathrm{m}$. Il est armé de deux couronnes de 11 à 17 crochets, soit un total de 22 à 34 . Les plus grands mesurent 27 à $32 \mu \mathrm{m}$ et les plus petits 23 à $27 \mu \mathrm{m}$. Les ventouses mesurent 65 à $75 \mu \mathrm{m}$ de diamètre. Les anneaux hermaphrodites mûrs, de 190 à $420 \times 210-220 \mu \mathrm{m}$, comptent 15 à 21 testicules en deux couches superposées, disposés en un champ unique contournant la glande vitellogène et remontant jusqu'en avant de la poche du cirre; cette dernière région peut comporter jusqu'à 2 ou 3 testicules. L'ovaire est massif, bilobé et réniforme, situé légèrement obliquement par rapport à l'axe du strobile et en arrière du milieu de l'anneau. L'oviducte est constitué d'un conduit de gros calibre formant d'abord oviscapte musculeux, puis courbé en $\mathrm{S}$ en arrière de l'ovaire; il reçoit le vitelloducte et le canal vaginal issu du réceptacle séminal. La glande de Mehlis se situe partiellement sous la glande vitellogène; celle-ci est massive, grande comme deux testicules environ et postérieure. Les pores génitaux sont irrégulièrement alternés et situés dans la moitié antérieure de l'anneau, de même que dans les anneaux gravides. La poche du cirre est globuleuse, relativement longue, atteignant le plus souvent 


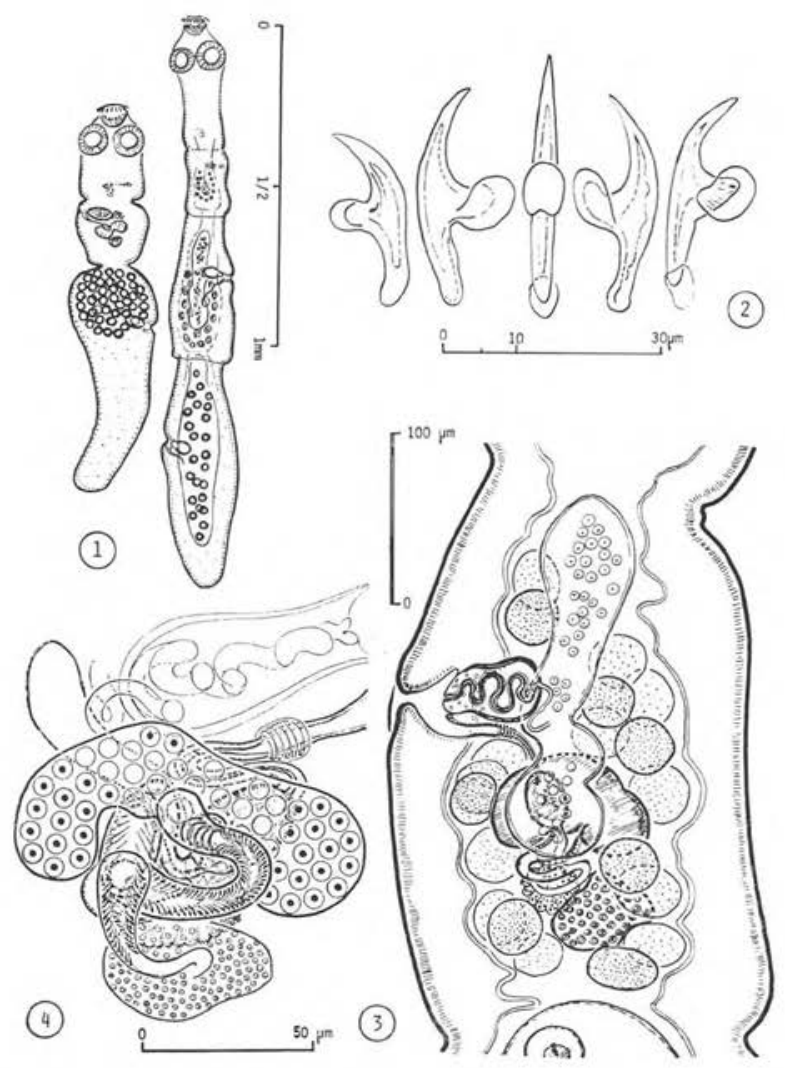

Fig. 6. Echinococcus multilocularis. 1. Cestodes adultes gravides. 2. Crochets du rostre (face et profil). 3. Anneau mûr. 4. Appareil reproducteur femelle (détail).

l'axe longitudinal de l'anneau. Le vagin est postérieur à la poche du cirre et présente un sphincter musculeux bien dessiné. Le canal vaginal est court. Les anneaux gravides sont de taille variable selon l'état de leur maturité $(480$ à $700 \mu \mathrm{m})$ et toujours plus courts que la moitié de la longueur totale du strobile. Ils ne contiennent que des glandes sexuelles involuées à l'exception des organes copulateurs, et un utérus sacculaire allongé dans le sens de l'anneau, et ayant tendance à pénétrer dans l'anneau antérieur adjacent. De rares strobiles offrent une image caractéristique d'utérus de contours circulaires, situé dans la moitié antérieure de l'anneau. L'œuf mûr, très généralement sphérique, et parfois ovalaire mesure $28 \times 32 \mu \mathrm{m}$ de diamètre, ou $39 \times 33$. Son enveloppe brune prismatique est épaisse de 5 à $4 \mu \mathrm{m}$. Le nombre des œufs mûrs dans l'utérus des anneaux gravides est extrêmement variable (de zéro à 40, parfois davantage dans quelques cas : 150-200). 
Discussion: La petite taille relative des crochets du rostre, le petit nombre de testicules distribués principalement en arrière de la poche du cirre, la position antérieure du pore génital, l'allure parfois typique des contours utérins par ailleurs non ramifiés, conduisent à la diagnose de l'espèce multilocularis de préférence à l'espèce granulosus. On sait d'expérience que le renard, sans être réfractaire à l'infestation par E. granulosus dans tous les cas, constitue à l'état naturel un hôte relativement défavorable de cette espèce de parasite. Enfin, la présence de l'espèce multilocularis chez les renards d'Auvergne paraît d'autant moins suspecte qu'un foyer actif d'échinococcose alvéolaire humaine a été décelé dans cette région géographique à partir de 1970, de part et d'autre de la limite des départements du Puy-de-Dôme et du Cantal.

Amoebotaenia paradoxa (Rud., 1802).

syn. : Amoebotaenia lumbrici (Villot, 1883) ; Sacciuterina paradoxa (Rud.) Matévossian, 1963.

3 hôtes parasités. Les strobiles longs de 1,5 à $2 \mathrm{~mm}$ et larges de 450 à $475 \mu \mathrm{m}$ ne comportent qu'une dizaine d'anneaux au maximum. Le scolex est large $(266 \mu \mathrm{m})$, aplati et court; il se confond postérieurement avec le cou. Il est muni de quatre ventouses inermes de $120 \mu \mathrm{m}$ de diamètre et d'un rostre puissant, évaginable à partir d'un étui, et armé d'une couronne unique de 12 à 14 crochets de 77 à $85 \mu \mathrm{m}$ de long. Leur silhouette est figurée (fig. 7).

Le strobile compte 3 ou 4 anneaux immatures, 3 ou 4 anneaux hermaphrodites mûrs et 2 ou 3 anneaux gravides. Les pores génitaux latéraux sont presque régulièrement alternés, et les canaux osmo-régulateurs (C.O.-R.) se situent ventralement par rapport aux conduits sexuels terminaux mâle et femelle. L'anatomie des anneaux hermaphrodites répond à Ja définition des Dilipididae. L'ovaire est linéaire, légèrement oblique, disposé transversalement en avant de l'anneau. Le vitellogène est massif, de la taille d'un à deux testicules et situé en arrière de l'isthme ovarien. Les testicules sont situés en deux couches superposées en arrière de l'ovaire; leur nombre est de 14 à 18. La poche du cirre est cylindrique, dépasse les C.O.-R. poraux, et sa longueur correspond au tiers environ de la largeur de l'anneau $(130 \times 23 \mu \mathrm{m})$. Le cirre invaginé présente une lumière tapissée de très fines épines courtes presque ponctiformes, sur une longueur de $75 \mu \mathrm{m}$. Le vagin est inerme et le canal vaginal est cerclé de fibres musculaires sur toute sa longueur, jusqu'au réceptacle séminal. L'anneau gravide de $520 \times 470 \mu \mathrm{m}$ montre un utérus sacculaire dépourvu de capsules utérines ou de capsules parenchymateuses. Le nombre des œufs est élevé; leur membrane externe est fine, lisse et transparente ; elle mesure $70-80 \times 45-50 \mu \mathrm{m}$; leur membrane interne est épaissie, colorable, et sa surface extérieure est finement gaufrée; elle mesure $35-38 \times 30-34 \mu \mathrm{m}$.

Discussion: Les traités classiques de Cestodologie ne mentionnent pas de Dilépididé chez les renards. Le cestode décrit, par son rostre à une seule couronne de 

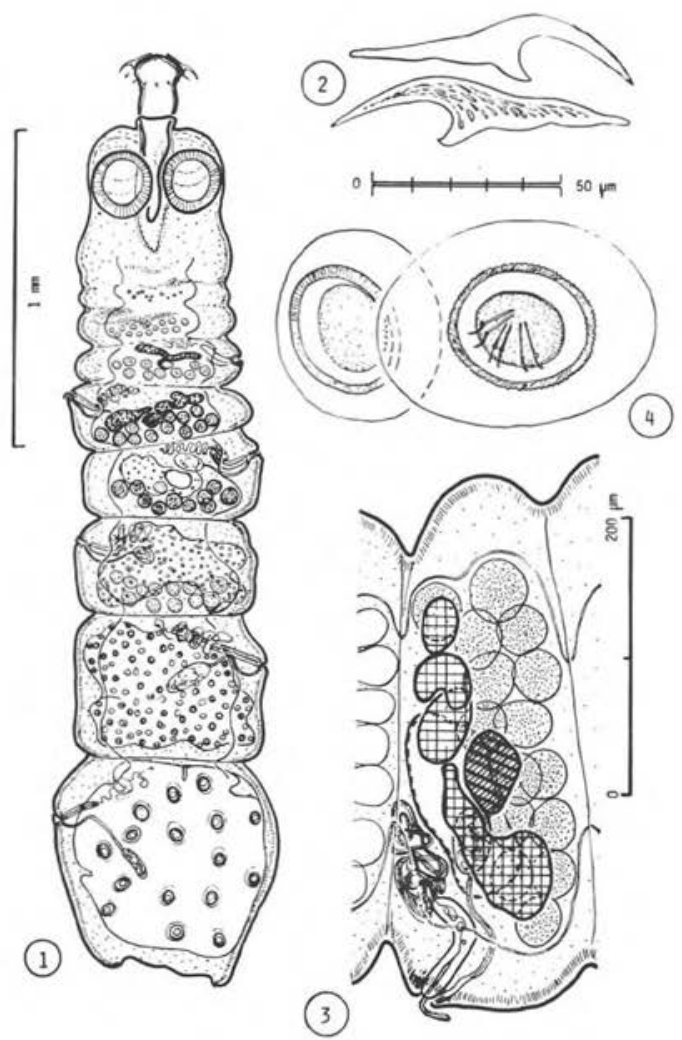

Fig. 7. Amoebotaenia paradoxa. 1. Cestode adulte complet. 2. Crochets du rostre. 3. Anneau mûr. 4. EEufs mûrs.

crochets, son strobile à nombre réduit d'anneaux, ses pores génitaux presque régulièrement alternés, ses conduits génitaux dorsaux par rapport aux C.O.-R. et son utérus sacciforme sans capsule, répond à la définition du genre Amoebotaenia Cohn, 1900 bien que ce genre soit considéré comme inféodé aux Oiseaux. Les crochets et l'anatomie correspondent à ceux de l'espèce paradoxa Rud., 1802. Si le renard constitue un hôte accidentel du ver [considéré comme un parasite habituel des Oiseaux Charadriiformes, notamment des Bécasses (Scolopax)], le cestode ne paraît pas souffrir de son hôte d'occasion puisqu'il n'y présente pas d'anomalie anatomique évidente et qu'il y devient fertile. (Deux hôtes toutefois présentent des strobiles immatures. Cette immaturité traduit soit une inadaptation hôte-parasite, soit une infestation trop récente pour avoir donné naissance à des adultes).

Cette découverte conduit à admettre que, au moins en Auvergne, A. paradoxa s'est adapté à deux hôtes définitifs éloignés zoologiquement : l'oiseau, hôte classique, 
et le mammifère, hôte vicariant. L'infestation de ces deux types d'hôtes se réalise par ingestion de vers de terre parasités.

Remarque: A. paradoxa constitue le type du genre Sacciuterina Matévossian, 1963 dont les pores génitaux sont définis comme irrégulièrement alternés. Cette irrégularité paraît assez accidentelle chez les cestodes de notre série. (Sur 41 strobiles dénombrés chez deux hôtes, 7 strobiles présentent chacun une fois la succession de deux pores génitaux du même côté, soit 7 anomalies sur un total de 243 anneaux successifs : 4 sur 28 strobiles du premier hôte et 3 sur 13 strobiles du second). C'est la raison pour laquelle l'espèce paradoxa est maintenue dans le genre Amoebotaenia dont les pores sont définis comme régulièrement alternés.

\section{II. - NEMATODES}

Les Nématodes parasites des renards se distribuent en six espèces différentes selon le catalogue suivant :

I. Famille des Ancylostomidae Lane 1917. Genre Uncinaria Froelich, 1789 : Uncinaria stenocephala (Railliet, 1884). 47 hôtes, soit $68,11 \%$ du total, hébergent une somme de 634 parasites (256 ơ et $378 q$ adultes). Leur nombre par hôte varie de 1 à 69.

Genre Ancylostoma (Dubini, 1843) ; A. caninum : 1 hôte, soit 1,44 \% du total, héberge 1 ver adulte + .

II. Famille des Trichostrongylidae Leiper, 1912. Genre Molineus Cameron, 1923 :

M. patens (Dujardin, 1845). 1 hôte, soit 1,44\% du total, hébergeant un couple de vers adultes.

III. Famille des Oxyuridae Cobbold, 1864.

Quelques femelles d'un genre non précisé chez un hôte, soit 1,44\% du total.

IV. Famille des Ascaridae Baird, 1853. 1) Genre Toxocara Stiles, 1905 : T. canis (Werner, 1782). 19 hôtes, soit 27,53 \% du total, hébergent une somme de 164 vers dont 146 adultes (66 $\sigma^{*}$ et 80 \%). Leur nombre par hôte varie de 1 à 24 . 2) Genre Toxascaris Leiper, 1907 : T. leonina (Linstow, 1902). 23 hôtes, soit 33,3 \% du total, hébergent une somme de 384 vers dont 376 adultes (182 $\sigma^{\star}$ et 194 \%) ; leur nombre par hôte varie de 1 à 150 .

\section{Les associations parasitaires.}

Le polyparasitisme constitue la règle générale chez les renards auvergnats. Le détail des associations parasitaires et leurs pourcentages sont énumérés dans le tableau $I$. 
Tableau I. Associations parasitaires.

\begin{tabular}{|c|c|c|c|}
\hline$\S$ & 69 hôtes & Nombre d'hôtes & Pourcentages \\
\hline \multirow[t]{3}{*}{1} & Renards: & & . \\
\hline & 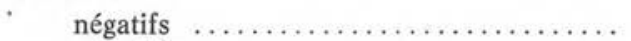 & 16 & 23,2 \\
\hline & 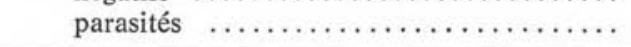 & 53 & 76,5 \\
\hline \multirow[t]{3}{*}{2} & Renards présentant : & & \\
\hline & des Cestodes ..... & 40 & 57,9 \\
\hline & des Nématodes $\ldots \ldots \ldots \ldots \ldots \ldots \ldots$ & 50 & 72,2 \\
\hline \multirow[t]{4}{*}{3} & Association : & & \\
\hline & Cestodes-Nématodes . & 37 & 53,6 \\
\hline & Nématodes seuls .................... & 13 & 18,8 \\
\hline & Cestodes seuls ..................... & 3 & 4,3 \\
\hline \multirow[t]{4}{*}{4} & Dans le cas des Cestodes, sont présents : & & \\
\hline & une espèce unique $\ldots \ldots \ldots \ldots \ldots$ & 25 & 36,2 \\
\hline & deux espèces différentes $\ldots \ldots \ldots \ldots \ldots \ldots$. & 11 & 15,9 \\
\hline & trois espèces différentes $\ldots \ldots \ldots \ldots \ldots \ldots$ & 4 & 5,8 \\
\hline \multirow[t]{4}{*}{5} & Dans le cas des Nématodes, sont présents: & & \\
\hline & une espèce unique $\ldots \ldots \ldots \ldots \ldots \ldots$ & 13 & 15,9 \\
\hline & deux espèces différentes ................ & 32 & 46,4 \\
\hline & trois espèces différentes $\ldots \ldots \ldots \ldots \ldots \ldots$. & 5 & 7,2 \\
\hline \multirow[t]{5}{*}{6} & $\begin{array}{l}\text { Dans le cas des associations Cestodes-Néma- } \\
\text { todes, sont présents: }\end{array}$ & & \\
\hline & deux espèces de parasites $\ldots \ldots \ldots \ldots \ldots$ & 11 & 15,9 \\
\hline & trois espèces de parasites $\ldots \ldots \ldots \ldots \ldots$ & 18 & 26,1 \\
\hline & quatre espèces de parasites ........... & 12 & 17,4 \\
\hline & cinq espèces de parasites .............. & 4 & 5,8 \\
\hline
\end{tabular}

\section{REMARQUES ET DISCUSSION}

La distribution géographique des parasites du renard ne montre pas de zones préférentielles de leur répartition, même s'il se présente quelques aires restreintes dans les deux départements du Puy-de-Dôme et du Cantal d'où les parasites les plus fréquemment rencontrés au cours de l'enquête apparaissent absents. Les lacunes décelables de cette répartition pourraient avoir plusieurs causes possibles : $a$ ) des autopsies trop peu nombreuses pour constituer un tableau fidèle de l'endémie parasitaire des hôtes de l'aire considérée ; $b$ ) une enquête trop restreinte dans le temps pour tenir compte des fluctuations annuelles de l'endémie parasitaire, sous la dépendance des conditions climatiques, des migrations des hôtes, ou de la biologie des divers hôtes des cycles évolutifs et de celle des parasites. 
Les observations concernant $E$. multilocularis sont encore trop peu nombreuses à l'heure actuelle et trop ponctuelles pour fournir des critères d'appréciation convenables concernant les variations saisonnières de l'endémie animale de cette parasitose en France. Les huit renards du Nord-Est du territoire national autopsiés par Coudert et coll., 1970 et trouvés porteurs du parasite sur un total de 167 hôtes, ont tous été autopsiés l'hiver (février-mars). Peut-être ne s'agit-il que d'une coïncidence, car les cinq renards positifs de notre série se distribuent sur trois saisons, hiver exclu (avril, mai, septembre, décembre). Compte tenu de la longévité courte du cestode adulte chez l'hôte définitif ( 3 à 4 mois), les microtidés hôtes intermédiaires devraient demeurer infestants en Auvergne toute l'année durant. Les mouvements migratoires de la population vulpine européenne, peut-être accélérés par sa destruction systématique dans certains de nos départements dans un but de prophylaxie anti-rabique, pourraient être à l'origine de l'implantation récente, mais apparemment solide, du foyer auvergnat d'échinococcose multiloculaire.

REMERCIEMENTS. - Nous tenons à remercier la Présidence de la Fédération des Chasseurs du Puy-de-Dôme (Président Dupic - Garde fédéral Schaeffer) et celle des Lieutenants de Louveterie du Cantal (Président Sarret), ainsi que les personnels respectifs de ces organismes. Sans leur collaboration efficace et dévouée pour la capture, la conservation et l'expédition des renards, aucune enquête parasitologique n'aurait pu être entreprise. Par ailleurs, $\mathrm{M}^{\mathrm{me}}$ Durette-Desset du Muséum d'Histoire naturelle de Paris nous a été d'une aide précieuse dans la détermination des Nématodes. Nous la remercions de sa compétence et de sa patience.

\section{Bibliographie}

Coudert J., Euzéby J., Garin J.-P.: Fréquence de E. multilocularis chez le renard commun (Vulpes vulpes) dans le secteur nord-est de la France. Lyon Méd., 1970, 224, 293-298.

Rey M., Morin B., Pétavy A.-F.. Cambon M., Baril A.: Premières observations auvergnates d'échinococcose alvéolaire. Nouv. Presse Méd., 1977, 6, 4 070-4 071. 